\title{
Measurement and analysis of reversible transformations of vortex-free and singular light fields produced by PDLC cells
}

\author{
V.V. Ponevchinsky, V.I. Vasil'ev, M.S. Soskin \\ Institute of Physics, National academy of Sciences of Ukraine, \\ 46, Prospect Nauki, 03028 Kyiv, Ukraine \\ Corresponding author: Phone +380445255566, Fax +380445251589, \\ Email:marat.soskin@gmail.com
}

\begin{abstract}
In this work, we have firstly realized and investigated reversible transformations of vortex-free and singular optical complex landscapes appearing in a He-Ne laser beam transmitted through a PDLC cell, which is controlled by an applied DC voltage. Biaxes of all micro size LC domains are oriented approximately along the applied electric field, and vortex-free complex landscape exists at $200 \mathrm{~V}$. At $160 \mathrm{~V}$, biaxes orientation spreads, and the first 'embryo' of vortices in the form of a smooth phase step appears. The first vortex pair nucleates at $140 \mathrm{~V}$. Multitude of vortex pairs is realized when electric field is switched off. Reversed evolution is realized when the applied DC voltage grows from zero value to $200 \mathrm{~V}$. It was shown that the observed topological evolution is fully defined by changes in structure and localization of real and imaginary zero-intensity lines for output complex light field. The developed technique of parametric dynamics can be applied successfully to realize and investigate various types of controlled optical elements including media with nanoadditions.
\end{abstract}

Keywords: singular optics, optical vortices, topology, PDLC cell.

Manuscript received 17.03.09; accepted for publication 14.05.09; published online 15.05.09.

\section{Formulation of the problem}

History of optical singularities, or wavefront dislocations, started in 1974 by the seminal J. Nye and M. Berry paper 'Dislocations in wave trains' [1]. It initiated creation of singular optics [2, 3] developing rapidly last two decades. Generic transformation of a smooth (vortex-free) complex landscape to a singular wavefront is the problem of principle importance for singular optics and singular wave physics at whole. It has not been resolved and even formulated up to now, due to our knowledge. For the beginning, existence of an inhomogeneous vortex-free scalar/elliptic field (speckle patterns) has to be justified in principle. Arbitrary complex landscape possesses the following general form [1]:

$$
\begin{aligned}
& E(x, y)=\rho(x, y) \exp (i \varphi(x, y))= \\
& =\operatorname{Re}[E(x, y)]+i \operatorname{Im}[E(x, y)] .
\end{aligned}
$$

Vortices appear at the crossing points of real $\operatorname{Re}[E(x, y)]=0$ and imaginary $\operatorname{Im}[E(x, y)]=0$ nodal lines of this landscape where the field amplitude is exactly zero, what causes the phase uncertainty [1-3]. In a vortex- free complex landscape, these nodal lines have to avoid each other. In a complex landscape with an irregular structure, 'percolating nodal lines meander back and forth and it is not easy to imagine any mechanism by which they can avoid each other' [2]. Therefore, M. Berry has constructed the exceptional complex landscape with a system of smooth symmetric amplitude hills, the zero average wavevector $\boldsymbol{k}$ and circular not intersecting system of nodal lines $\operatorname{Re}[E(x, y)]=0$ and $\operatorname{Im}[E(x, y)]=0$, which are shifted [2]:

$$
\operatorname{Im}[E(x, y)]=\operatorname{Re}[E(x-1 / 2, y-1 / 2)],
$$

Such type vortex-free complex landscapes can't be transformed to a vortex complex landscape. Therefore, our first task to be solved from the outset was finding of a real controlled media with a vortex-free complex landscape in a transmitting laser beam. Photorefractive lithium niobate crystals used in all our investigations of dynamic singular optics [5-9] would not do because output scattered speckle field possesses many singularities at the very beginning due to unavoidable random refractive index heterogeneities. A managed 


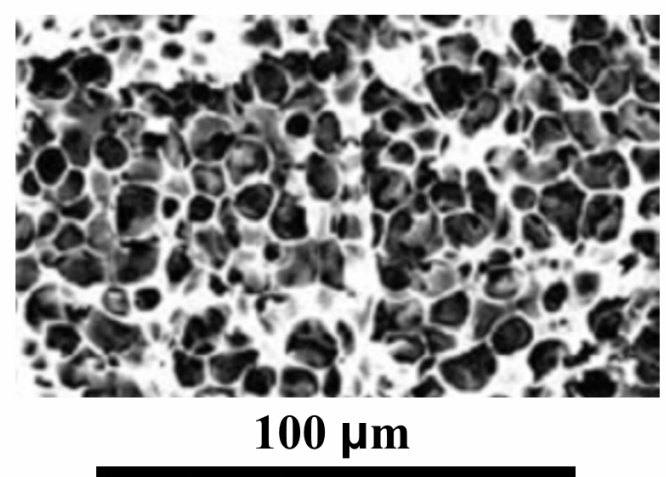

(a)

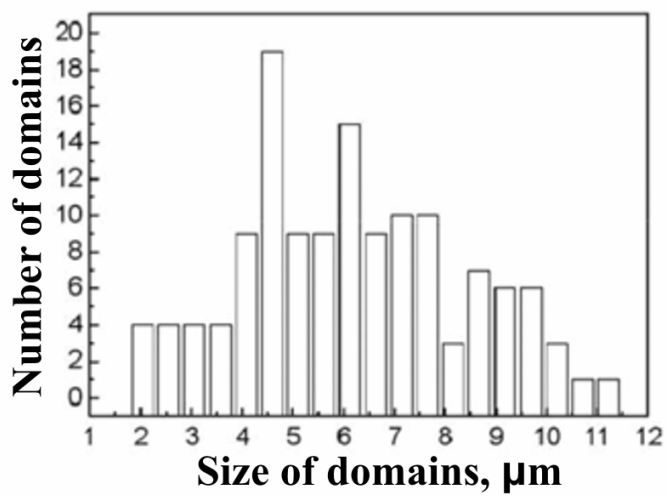

(b)

Fig. 1. Polymer morphology of the PDLC composite with 35 vol\% of a polymer phase. (a) Scanning electron microscope (SEM) image of the polymer phase; $(b)$ diagram of the size distribution of liquid crystals (LC) domain voids. (Courtesy of [10] co-authors).

nonlinear medium is suited, which allows to transform nodal line up to their intersection needed for optical vortices creation [5].

The plan of paper is as follows. The structure and parameters of the used electrically controlled PDLC cell are described in Sec. 2. Sec. 3 presents the elaborated technique for amplitude and phase distribution measurements in an output beam cross-section, procedure of data processing. Sec. 4 considers evolution of the complex landscape from vortex-free to multiplevortices complex landscape with diminishing value of applied DC electric field. For understanding the mechanism of a vortex-free complex landscape transformation to a singular wave front, statistics of an intensity distribution was measured as a function of the applied electric field value. Obtained results and general questions of topological transformation of a vortex-free complex landscape to the singular complex landscape and vice versa are discussed. The main findings are summarized in Sec. 5.

\section{Electrically controlled PDLC cells}

We have focused on polymer-dispersed liquid crystal (PDLC) cells with optical properties controlled by applied DC electric field ([10] and literature therein). The PDLC cell with $20 \mu \mathrm{m}$ thickness and $40 \mathrm{vol} \%$ of polymer (the nematic mixture E7 from Merk) was used. Polymer matrix of such concentration possesses a sponge-like structure (Fig. 1a) with $6 \mu \mathrm{m}$ average size of LC domains voids (Fig. $1 b$ ). Therefore, a propagating beam interacts with 3 to 4 layers of LC voids. The refractive index of the used polymer was intermediate between the liquid crystal ordinary and extraordinary refractive indices of nematic mixture E7 from Merk.

Biaxial axes of LC domains are directed randomly without applied electric field. Therefore, an incident light beam scatters strongly and cell transparency is nearly zero [10]. Applied electric field with strong enough DC voltage aligns biaxial axes of LC domains along the direction of electrical field with small deviations. Its value is specific for each PDLC cell and was $200 \mathrm{~V}$ in our case. As a result, the cell transparency grows strongly. Due to this property, PDLC cells are used nowadays in various optical systems as an element with controllable transparency [10]. We have investigated firstly PDLC cells from the viewpoint of singular optics as element suitable for controlled parametric dynamics of light fields with various complex landscapes. The idea was clear and simple. We were waiting that controllable transition from light scattering regime to transparent state of a cell controlled by the value of applied DC voltage could be followed by essential change of output beam singular properties. And obtained results live up to our expectations.

\section{Measurement technique and data processing}

The experimental setup is shown in Fig. 2. The selected plane of a measured fragment of an investigated complex landscape was projected on a CCD camera with $10^{\times}$magnification. Its $15-\mathrm{cm}$ distance from the PDLC cell what was enough for overlapping and interference of scattered light components. The typical angle between object and reference beams was $\sim 0.01 \mathrm{rad}$ to obtain interferential patterns with $\sim 80 \mu \mathrm{m}$ fringes.

Interference of a speckle field with plane wave rises to the intensity pattern $I(x, y)$ :

$$
\begin{aligned}
& I(x, y)=a(x, y)+b(x, y) \times \\
& \times \cos \left(k_{x} x+k_{y} y+\varphi(x, y)\right),
\end{aligned}
$$

where $a(x, y)$ is an interference background component, $b(x, y)$ is an interference signal envelope, $\varphi(x, y)$ is a speckle field phase distribution, $k_{x}$ and $k_{y}$ are spatial frequencies caused by a relative tilt of interfering beams. We use the modified method of Fourier-transform to 


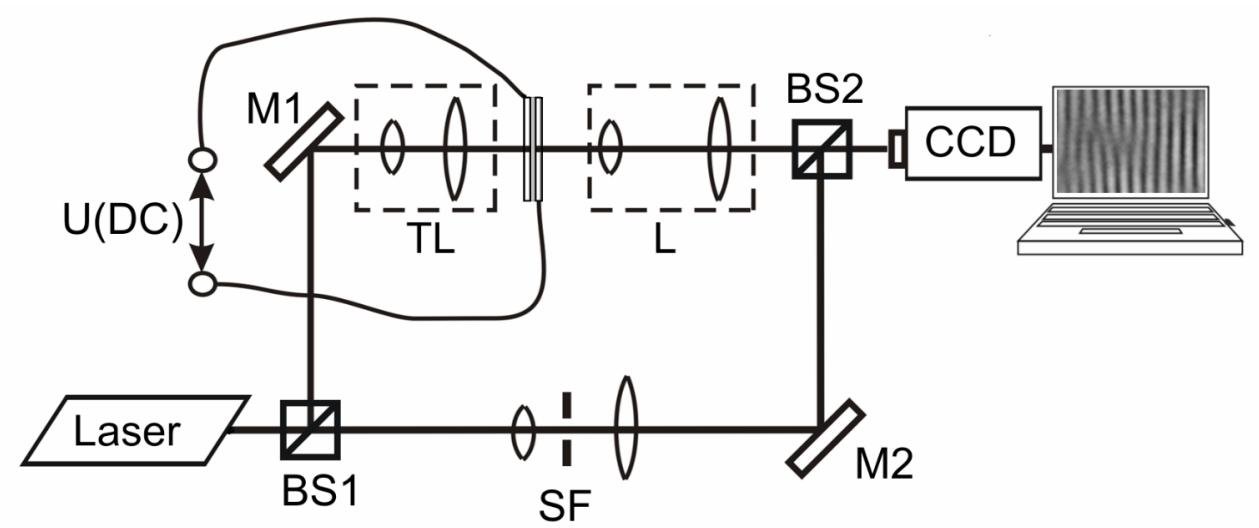

Fig. 2. Experimental setup. Beam splitters BS1, BS2 and mirrors M1, M2 form a Mach-Zehnder interferometer tuned to obtain a finite fringe interferogram. Linearly polarized Gaussian beam of He-Ne laser is directed on the entrance plane of splitter BS1. Interference patterns are recorded by the digital camera FINE T-392C with $720 \times 576$ pixels screen. A PDLC cell is placed into an object arm of the interferometer. A telescope TL expands object beam up to $2 \mathrm{~mm}$ diameter. A spatial filter SF forms plane wave in the reference beam. When the reference beam is shut off, the setup is used for acquisition of intensity distribution. The lens system L projects appeared speckle field in a selected plane onto the screen of the CCD camera.

reconstruct phase distribution from the interference pattern bitmap [11]. To correctly run the algorithm, we need the interference fringe width to be a multiple of the used CCD camera pixel size. For any field fragment with optical vortex,

$$
\oint_{C} d \varphi(x, y)=2 \pi
$$

The path of integration $C$ is a boundary of a square that is usually taken to be 2 pixels on a side.

The measured intensity distribution $I(x, y)$ and the reconstructed phase map $\varphi(x, y)$ give full information about the investigated speckle field $E(x, y)$ with real and imaginary parts

$$
\left\{\begin{array}{l}
\operatorname{Re}[E(x, y)]=\sqrt{I(x, y)} \cos (\varphi(x, y)) \\
\operatorname{Im}[E(x, y)]=\sqrt{I(x, y)} \sin (\varphi(x, y))
\end{array},\right.
$$

Zero lines and discrete equiphase contours for the measured fragment of an output beam complex landscape allow to fix arrangement of all existing singularities and embryos of potential singularities. Indeed, the phase interval between neighbor nodal lines $\operatorname{Re}[E(x, y)]=0$ and $\operatorname{Im}[E(x, y)]=0$ is $\pi / 2$ with the averaged phase gradient

$$
\left\langle\frac{d \varphi}{d r}\right\rangle=\frac{\pi}{2 \Delta r}
$$

where $\Delta r=\left[\left(x_{1}-x_{2}\right)^{2}+\left(y_{1}-y_{2}\right)^{2}\right]^{1 / 2}$ is the minimal distance between analyzed nodal lines. This phase gradient tends to infinity when they approach. Arrangement of all the existing vortices into topological network [12, 13] can be established, if saddles and traverse them equiphase lines starting and finishing on the vortices are found from the measured interference map.

\section{Results and discussion}

According to the principle of PDLC cell functioning, the control parameter is value of applied DC voltage which tries to arrange biaxes along the electric field direction. The full gamut of complex landscapes from vortex-free at high enough DC voltage up to its absence when complex landscape possesses multi-vortices generic (natural) form was realized and measured. The applied voltage range was 0 to $200 \mathrm{~V}$. For each voltage, we have measured crucial characteristics of a complex landscape: phase map and contours of real $\operatorname{Re}[E(x, y)]$ and imaginary $\operatorname{Im}[E(x, y)]$ zero lines. The experiment has shown that it's enough to trace equiphase contours with $\pi / 16$ interval to find all actual topological features of a complex landscape. The characteristic complex landscape structures and corresponding values of applied voltages were chosen (Fig. 3): (a) the vortex-free complex landscape $(200 \mathrm{~V})$, (b) the first nearly touch of nodal lines, i.e. developed vortex 'embryo' as for polarization singularities [6] $(160 \mathrm{~V})$, (c) nucleation of the first vortex pair $(140 \mathrm{~V})$, and $(\mathrm{d})$ initial generic field at switched off electric field.

The evolution of an output beam complex landscape is defined by growing random orientation of biaxes directions resulting in speckle patterns structuring with development of deeper intensity minima. Full destructive interference is needed for complete destructive interference in the points where vortices appear [14]. In turn, this can happen for fully random amplitude and phase distribution in the field local structure of complex landscape around this point [6]. To understand better the matter of heart of the complex landscape topological evolution, we have measured statistics of speckle patterns intensity distribution in output speckle patterns at values strengths of the applied field through 20 independent realizations for each 
measured point (Fig. 4). The light field statistic after a gaussian diffuser is shown as the limited case measured recently for speckle patterns generated by lithium niobate crystal [9]. It possesses the negative exponential distribution [15]:

$$
p(I)=\frac{1}{\langle I\rangle} \exp \left(-\frac{I}{\langle I\rangle}\right),
$$

Let us consider now measured topological evolution of the complex landscape structure and corresponding statistics of the speckle pattern intensity as a function of the applied electric field. At the strong field $(200 \mathrm{~V})$ (Fig. 3a), biaxes of all LC domains are oriented along the direction of electric field with small deviation. Therefore, full destructive interference is absent automatically and the complex landscape is vortex free. Its intensity statistics differs cardinally from gaussian diffuser statistics and has the form of low broad curve with zero probability density at zero intensity (Fig. $4 a$, curve 1 ). It corresponds to intensity distribution of a weak modulated speckle patterns. The phase map is also smooth enough with few smooth phases extrema. It is natural, that complete destructive interference is impossible. Two real and imaginary zero lines are fully separated and meander in approximately the same averaged direction for our generic/natural complex landscapes contrary to M. Berry apprehension [2].

Contrary to smoothness of intensity distribution, there are the distinguished small areas where angular distribution of LC domain biaxes is much broader then in average. Namely, such situation is realized in right upper quadrant where two nodal lines $\operatorname{Re}[E(x, y)]=0$, $\operatorname{Im}[E(x, y)]=0$ approach essentially with the highest phase gradient between them among the shown phase landscape. This corresponds to the phase fold with $\pi / 2$ as vortex embryo in full analogy with measured recently azimuth step in random elliptic speckle patterns as the embryo of $\mathrm{C}$ point pair nucleation [6]. Therefore, this pair of neighbor nodal lines is a promising candidate for vortex creation at lower voltages of electric field.

Descent of the applied field up to $160 \mathrm{~V}$ leads to spread of biaxes orientation. Destructive interference grows and amplitude minima deepen. Most of isolated $\operatorname{Re}[E(x, y)]=0 \quad$ loops append to $\operatorname{Re}[E(x, y)]=0$ augment its meandering contrary to very small changes of $\operatorname{Im}[E(x, y)]=0$ contours (Fig. $3 b$ ). This is natural because the speckle pattern structure is mainly sensitive to statistics of biaxes distribution. Real and imaginary zero lines near the existing singularity embryo become close and almost tangent, which strongly enhances the embryo phase gradient tend to infinity, what corresponds to the threshold value of the vertical $\pi / 2$ height step and

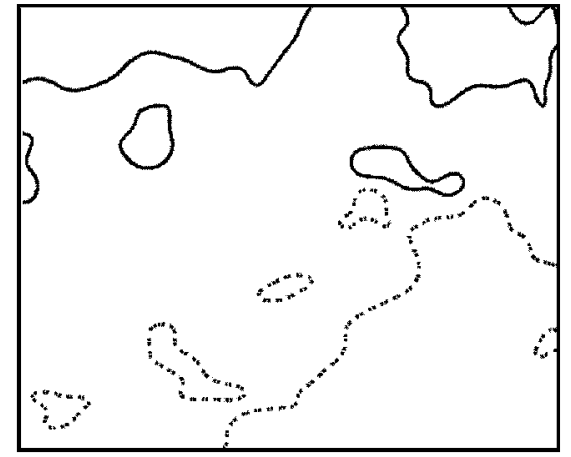

(a)

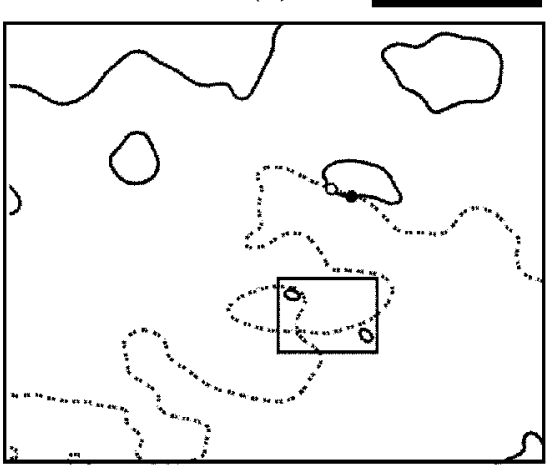

(c)

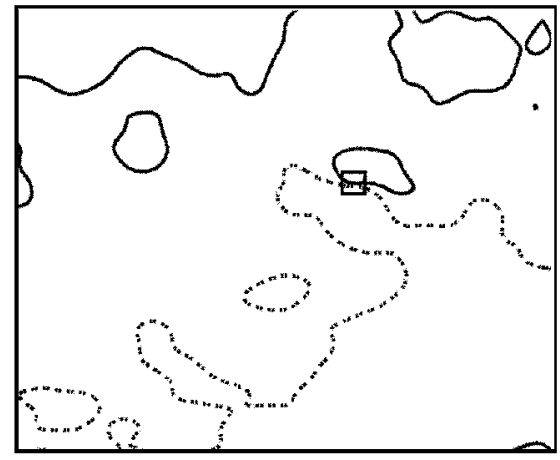

(b)

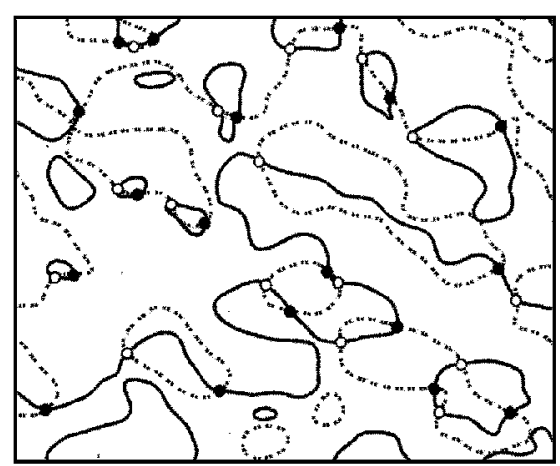

(d)

Fig. 3. Evolution of optical complex landscape induced by the PDLC cell. (a) Vortex-free landscape (200 V) (frame deposits the embryo); (b) Vortex-free landscape before first vortex pair creation (160 V) (frame deposits the pre-threshold embryo); (c) creation of the first vortex pair $(140 \mathrm{~V})$; (d) the initial complex landscape with vortices multitude (electric field is switched off). Positive (negative) charged vortices are white circles with black rimes (black circles). Nodal lines of $\operatorname{Re}[\mathrm{E}(\mathrm{x}, \mathrm{y})] / \operatorname{Im}[\mathrm{E}(\mathrm{x}, \mathrm{y})]$ are shown bold/dashed. 
wavefront tier. When the electric field is above $140 \mathrm{~V}$ the embryo transforms to a vortex pair (Fig. 3c), which indicates full destructive interference in vortex centers. Two additional embryos appear simultaneously below this vortex pair, what prepares nucleation of two new vortex pairs. But creation of the vortex pair can't change essentially the $p(I)$ curve. Indeed, it moves a little to the lower intensities and its maximum grows slightly (Fig. 4, curve 2).

The first quadruple of vortices appears at $70 \mathrm{~V}$, statistics curve prolongs to move to lower intensities, and its maximum grows (Fig. 4, curve 4). Finally, multitude of vortices exists without applied field (34 OVs in checked field fragment), i.e. at not perturbed PDLC cell (Fig. $3 d$ ). The $p(I)$ curve shifts to low intensities, its half-width diminishes and maximum grows essentially. Of course, it differs from true Gaussian curve. It witnesses that LC domains in a PDLC cell don't possess full random statistics. The careful reader will fix essential change of topology of a generic not perturbed complex landscape. Besides seen above crossing closed contours, there are two separated meandering nodal lines $\operatorname{Re}[E(x, y)]=0$, $\operatorname{Im}[E(x, y)]=0$ that are stud with contours of other sorts. The meander zero lines don't intersect.

It was known that in singular vector fields two near located $\mathrm{C}$ point dipoles as in Fig. $5 a$ are self-organized spontaneously in quadruples with both pairs of equal sign $\mathrm{C}$ points connected pairwise by equiazimuth lines through an open saddle [16]. It was natural to expect that this property is valid for scalar optical fields, too. Our first measurements have confirmed existence of such quadruples in fields after a PDLC cell [9]. Investigations undertaken this time allow to obtain a detailed structure of there quadruples (Fig. 5b).
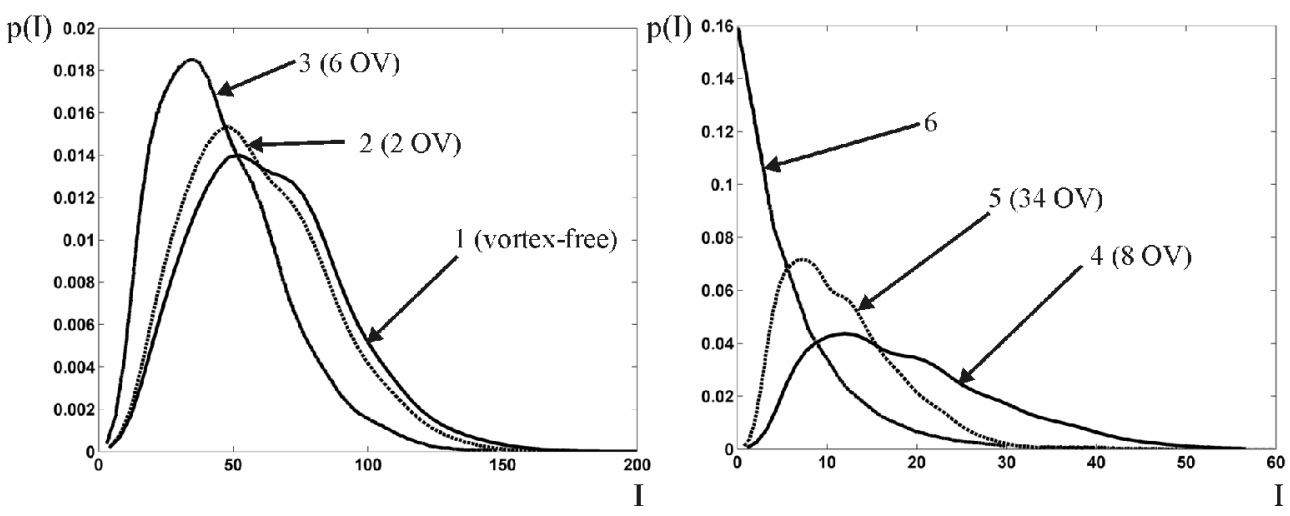

Fig.4. Measured statistics of the speckle pattern intensity (arbitrary units) distribution governed by the voltage of electric field applied to the PDLC cell: $1-200 \mathrm{~V} ; 2-140 \mathrm{~V} ; 3-100 \mathrm{~V} ; 4-70 \mathrm{~V} ; 5$ - switched-off applied field; 6 - the intensity distribution statistics of light field after a gaussian diffuser.

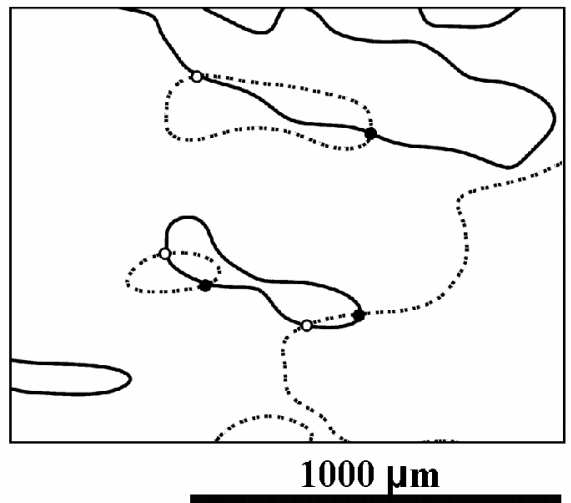

(a)

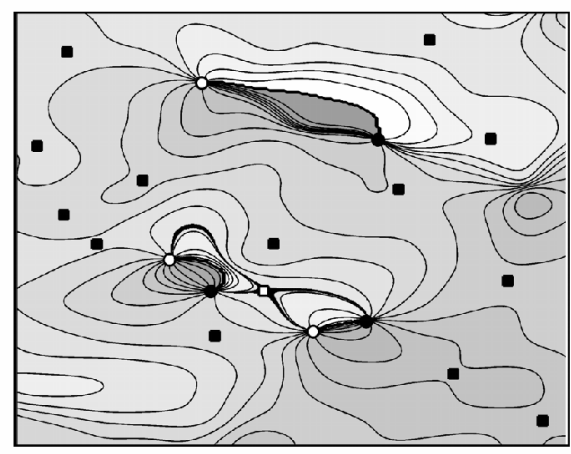

(b)

Fig. 5. Self-organization of nucleated vortex dipoles to the first quadruple $(70 \mathrm{~V}) . a$ - Complex landscape at $70 \mathrm{~V}$ with the first vortex quadruple nucleated. The nodal lines of $\operatorname{Re}[\mathrm{E}(x, y)] / \operatorname{Im}[\mathrm{E}(x, y)]$ are shown bold/dashed. The positive (negative) charged vortices are white circles with black rimes (black circles). The first vortex quadruple is shown in the black frame. $b$ - Full phase map with saddles (black squares) and equiphase lines shown with $\pi / 16$ interval. The bold equiphase lines connect the equal-charged vortices through the open saddle, that is shown by white square. All other squares are shown by black squares. 
Presented results show an essential difference of real-time and parametric dynamics of random fields. The real-time dynamics contains sequence of local creation and annihilation events happen during field development by chain and loop topological events [6]. Contrary, one-direction variation of the control parameter in the parametric dynamics produces separately nucleation and annihilation processes as we have seen for PDLC cells. Nevertheless, all the observed topological events are fully reversible when the applied DC voltage diminishes instead of grow.

Topology laws are valid for all systems. Therefore, the presented results have to be valid for all heterogeneous non-stationary systems with topological defects. They open wide possibilities for application of singular optics technique for investigation of various physical, chemical, etc. systems controlled by some parameter (temperature, composition, concentration of special additive, etc.). Optical vortex core is mathematical point/line in $2 \mathrm{D} / 3 \mathrm{D}$. Therefore, singular optics opens unique possibilities for investigation of nanosystems of various origins.

\section{Conclusion}

1. PDLC cells controlled by applied DC electric field have shown manageable reversible transition from a vortex-free complex landscape of a transmitted $\mathrm{He}-\mathrm{Ne}$ laser beam at high voltages to a multiple-vortices complex landscape without applied field and vise versa. To our knowledge, PDLC cells are the first class of topologically controlled condensed media that provide creation of optical singularities.

2. A vortex-free complex landscape initiated by a PDLC cell at high applied DC electrical field transforms to a singular complex landscape by complete destructive interference in seldom areas where singularity pairs are created. This happens due to transformation of LC domains biaxes oriented along a strong enough applied DC field to their fully disordered orientation in the vicinity of nucleated optical vortices at lower voltages.

3. It was established a detailed scenario creation of the first singularity pair through evolution of a created high phase gradient structure as 'embryo' up to the threshold value of a control parameter. The same scenario is valid for creation of all the following singularities at other values of the control parameter.

4. The developed technique of parametric dynamics can be applied successfully for realization and investigation of various types of controlled optical elements including those with nanoadditions.

\section{Acknowledgement}

This work was supported by STCU Project 4687. Authors are thankful to Drs. L.A. Dolgov, O.V. Yaroshchuk for provision of PDLC cells, introduction to their physics and numerous fruitful discussions. Authors are thankful to Prof. M. Berry (Bristol University) for fruitful discussions.

\section{References}

1. J. F. Nye and M.V. Berry, Dislocations in wave trains // Proc. R. Soc. Lond. A. 336, p. 165-190 (1974).

2. M.V. Berry, Vortex-free complex landscapes and umbilic-free real landscapes // J. Phys. A: Math. Theor., 40, p. F185-F192 (2007).

3. J. F. Nye, Natural Focusing and Fine Structure of Light. Institute of Physics, Bristol, 1999.

4. M. S. Soskin, M. V. Vasnetsov, Singular optics // Progr. Opt., 42, p. 219 - 276 (2001).

5. V.I. Vasil'ev, M.S. Soskin Analysis of dynamics of topological peculiarities of varying random vector light fields // Ukr. J. Phys., 52, p. 1123-1129 (2007).

6. V. Vasil'ev and M. Soskin, Topological and morphological transformations of developing singular paraxial vector light fields // Opt. Commun., 281, p. 5527-5540 (2008).

7. V.I. Vasil'ev and M.S. Soskin, Topological scenarios of the creation and annihilation of polarization singularities in non-stationary optical fields // JETP Lett., 87, p. 83-86 (2008).

8. V.I. Vasil'ev, M.S. Soskin, Dynamic singular optics // in:Complex Light and Optical Forces II, Proc. SPIE, 6905, p. 690505-1-6905-9 (2008).

9. M.S. Soskin, V.I. Vasil'ev, V.V Ponevchinsky, Chain topological reactions in developing random light fields // in: Complex Light and Optical Forces III, Proc. SPIE, 7227,7227-0.

10. L. Dolgov, O. Yaroshchuk, L. Qiu, SEM investigations of the polymer morphology in the liquid crystal-polymer composites with different polymer content // Mol. Crysr., Liq. Cryst., 468, p. 335-344 (2007).

11. Mitsuo Takeda, Hideki Ina, and Seiji Kobayashi, Fourier-transform method of fringe-pattern analysis for computer-based topography and interferometry // J. Opt. Soc. Am., 72, p. 156-160 (1982).

12. V.G. Denisenko, M.S. Soskin, and I. Freund, Optical polarization singularities and elliptic stationary points // Opt. Lett., 28, 1475-1477 (2003).

13. M. Soskin, R. Egorov, V. Denisenko, Topological networks of paraxial ellipse speckle-fields // J. Opt. A: Pure Appl. Opt., 6, S271-287 (2004).

14. M.V. Berry, Optical vortices // in: 'Optical vortices'. eds. Kestutis Staliunas and Mikhail Vasnetsov (Nova Science Publ., N.Y., 1999).

15. J.W. Goodman, Statistical properties if laser speckle patterns // in: Laser speckles and related phenomena, ed. J.C. Dainty, Berlin, Springer, 1975.

16. I. Freund, M. S. Soskin, A.I. Mokhun, Elliptic critical points in paraxial optical fields // Opt. Commun., 208, p. 223-253 (2002). 